\title{
The Design of Province-Region Distributed Supervisory Control System
}

\author{
YANG Jianping ${ }^{1}$, WANG Zhihua ${ }^{1}$, SUN Mingyang ${ }^{2, a}$, LIU Jin ${ }^{2, b}$, CHEN Hongfu ${ }^{1}$ \\ and ZHANG Yonggang ${ }^{2, c}$ \\ ${ }^{1}$ State Grid Shanghai Electric Power Company, Shanghai, China \\ ${ }^{2}$ China Electric Power Research Institute, Nanjing, China \\ asunmingyang@epri.sgcc.com.cn, ${ }^{b}$ liujin@epri.sgcc.com.cn, ${ }^{\mathrm{C}}$ zhangyongang@epri.sgcc.com.cn
}

Keywords: dispatching automation system; integration of provincial and districted dispatch; distributed supervisory and control system; wide area back-up

Abstract. Power grid dispatching automation system in future should follow the integrity nature of provincial level power grid. Since we consider provincial power grid as an organic whole, the real physical power grid should be described with a unified model, and its operation should be supported by a unified system. A unified system is not the one simply putting all the servers together, but the one with all infrastructures physically distributed. Nonetheless, dispatching decision applications should be centralized. That is to say, grid perception and real-time control are distributed physically, while analysis and decision are integrated logically. This paper proposed a design of distributed supervisory and control system with a unified model for its provincial and regional subsystems. It is expected that this architecture could provide a reference for others on the research, development and construction of power grid dispatching automation system.

\section{Introduction}

Considering the physical properties of power grid in china, provincial grid is an organic and indivisible whole. But to meet the needs of grid dispatching management, provincial grid is divided artificially into hierarchical grids, including province-level and region-level grid. Dispatching centers of two levels are setup accordingly in those regions. As a supporting tool, automatic system is also configured according this hierarchy. The dispatching automation system which has unified supporting platform and which follows standard IEC 61970, has been widely applied and played an important role in electric power dispatching operation $[1,2]$. The dispatching system configured according to geographical area, meets the need to hierarchy dispatching management, but violates the physical properties of electric power grid and impairs the abilities to make maximum use of grid resource. In order to improve the abilities of unified grid dispatching, lots of research and practice about the collaboration among dispatching automation systems have been carried out for many years. Though many great achievements have been made, multiple collaborative systems has less effect upon optimal operation of interconnected power grid than a single integrated system. Certainly, dispatching automation system configured based on geographical regions and hierarchy currently is a solution with respect to the limitations of IT technology.

In the future, provincial power grid should have one grid, one model and one system. Along with the rapid development of computer and network communication technology, it provides a possibility to make "one grid, one model, one system" come true. " One grid, one model, one system" doesn't means simply putting all the servers together to make up an independent system. The power grid of china has characteristics of significant grid level difference and wide geographical coverage. It is impractical to make grid dispatching system centralized like banking system in terms of power grid real-time operation. The infrastructure of future grid dispatching system must be distributed physically. Nonetheless, applications like network analysis should be configured centrally. Thus can help dispatching automation system improve the accuracy of judgement about grid operation status and the efficiency of dispatching decision. 
The feature of power grid dispatching automation system in the future is: grid perception and real-time control are distributed physically, analysis and decision of grid are integrated logically. Compared with existing smart grid dispatching and control system, the architecture of future system is founded on the current distributed structure in terms of voltage-grading and district-dividing, and it rounds up global applications of provincial dispatching system based on the needs of the whole grid dispatching business. It includes: (1) centralization of basic model data; (2) centralization of grid analysis application; (3) centralization of dispatching planning application; (4) centralization of real-time control decision. The supervisory and control system in each part of grid, including basic SCADA and real-time control, should be distributed to completely guarantee the real-time of grid perception and control. The business of supervisory and control system which is independent of global information can be processed locally and that embodies the principles of "centralized dispatching, distributed supervisory and control" [3].

\section{Overall Framework of Distributed Supervisory and Control System}

In order to achieve objectives of distributed real-time supervisory and control system, the design of the overall framework follows the principles of commonality, standard and openness. It introduces secure operating system and unified resource management, and hides the difference of underlying hardware for upper-layer application. In this way, unified task scheduling mechanism is provided. Also, uniform data reading and writing function is implemented by using the interface of the distributed real-time database to gather the results of data processing [4].

Fig. 1 shows the overall framework of distributed supervisory and control system. Supervisory and control centers of different level, including provincial and regional dispatching centers, are connected through WAN. SCADA systems using distributed technology are deployed in these dispatching centers. In this way, local grid real-time data is provided for local platforms and servers to support local applications including SCADA and AGC/AVC, etc. Thus, distributed monitoring, operation and control is realized, and data access service is available for upper-level dispatching centers.

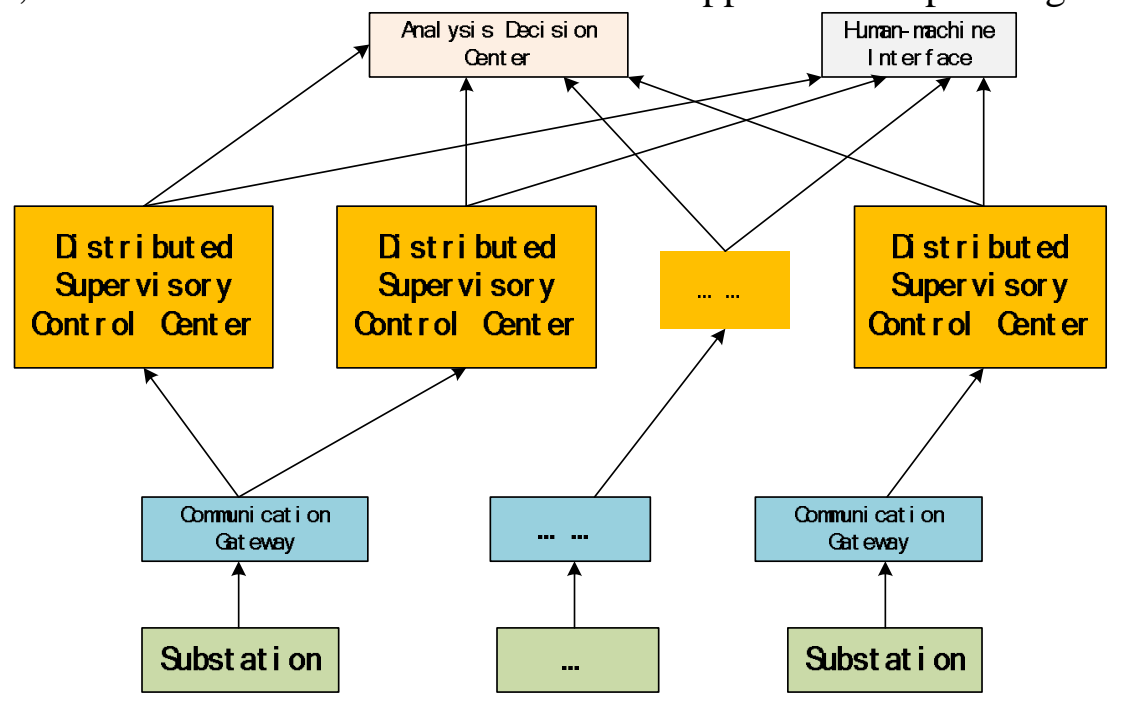

Fig. 1 overall framework of distributed supervisory and control system.

All the model of distributed supervisory control system only comes from model center to keep consistency of the whole system. As the data interchange link of the system, communication gateway processes the "four remotes" data, and then forwards the data to right center according to data forwarding rules. Analysis decision center and human-machine interface can get the grid real-time data from the distributed supervisory control centers through access interface of distributed real-time database.

Fig. 2 presents hardware architecture of distributed supervisory control center, including the aspects as follows: (1)Each of the supervisory control center consists of SCADA server cluster, WAMS server cluster, automation control server group, historical data server group, real-time data access server 
cluster; (2)SCADA server cluster includes data node server cluster and public node server cluster. The former is responsible for distributed processing of measuring data, and the latter is in charge of data calculation and analysis with all the model and data in the range of the supervisory control center. (3)Boundary data is interchanged between supervisory control centers. Measuring data and control command are interchanged between supervisory control center and integrated analysis decision center. Upstream data and downstream data are interchanged between supervisory control center and substation.

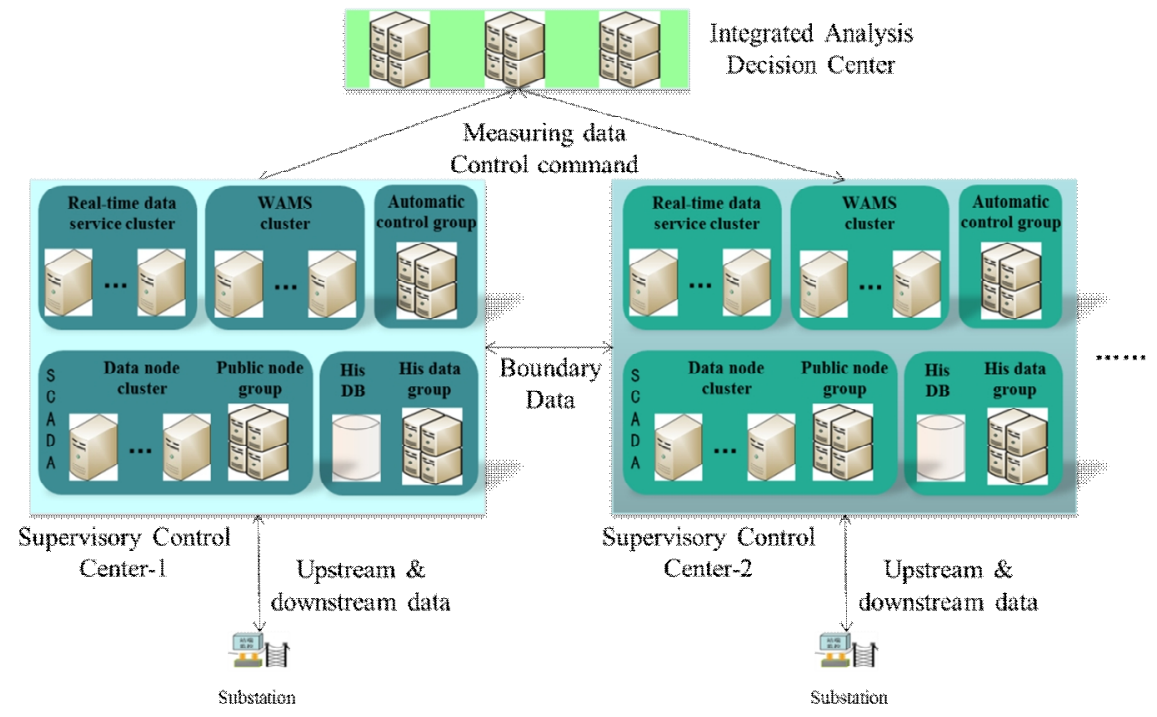

Fig. 2 Hardware architecture

Fig. 3 presents software architecture of distributed supervisory control center, including several aspects as follows: (1) Platform layer. The new generation of dispatch technical supporting system provides support for applications of distributed supervisory control center; (2) Cluster management layer. Most of applications in distributed control center are clustered. So this layer is so important and it includes resource management, task management and data synchronization; (3) Application layer. For SCADA, it contains data processing, calculation, topology coloring and so on. (4) Data access layer. It provides distributed data access service for upper-layer clients; (5) Clients layer. Clients include departments of dispatching, monitoring, mode, planning in the center as well as analysis decision center and other supervisory control centers which need the data here.

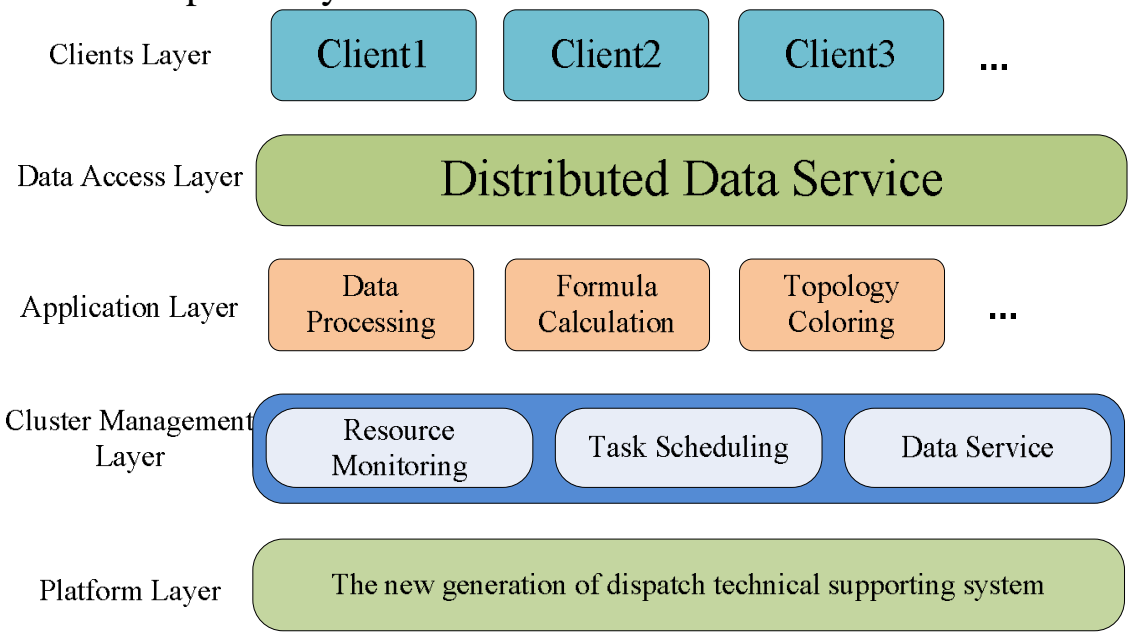

Fig. 3 Software Structure

\section{SCADA clustering mechanism}

Traditional dual real-time supervisory control servers are extended to a cluster in which there are two kinds of nodes, public node and data node. It can have two or more equal data nodes each of which downloads different grid model and takes the charge of data processing and UI accessing within model 
range. Data nodes update local real-time database and send the data to public node after processing the real-time data. The number of public nodes can be one or two. Public node downloads the whole grid model and has all real-time data. It's responsible for global data processing and UI accessing. The configuration of data division is made in communication gateway based on the distribution of model among data nodes in the cluster. Communication gateway and data nodes contact with each other through message bus. Clients and application servers get data from data nodes or public node in the way of load balancing through distributed data accessing [5-7].

SCADA clustering diagram is shown in Fig. 4. Instead of dual servers in active-standby mode before, multiple servers get together to process real-time data concurrently in cluster. Servers are divided into data node and public node. Measuring data is stored separately among data nodes, while device data is only stored in public node. So that measuring and device data can be decoupled clearly to support parallel computing.

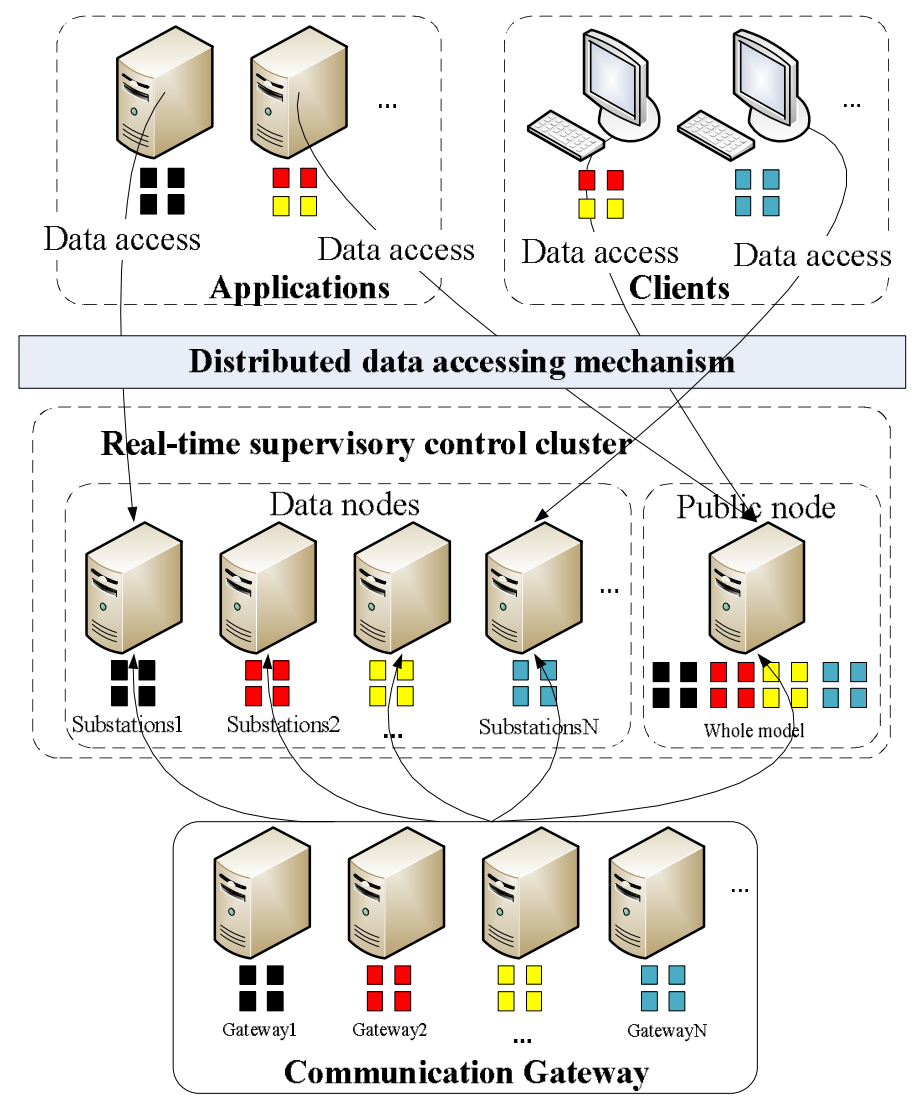

Fig. 4 SCADA clustering diagram.

\section{Design of Typical SCADA Functions}

The typical functions of SCADA include data processing, formula calculation and topology coloring. Overall framework of new generation of supervisory control system is designed with distributed technology over wide area, and the mechanism of SCADA cluster is applied in distributed supervisory control center. That's why the typical functions of SCADA need to be redesigned to support SCADA cluster and distributed monitoring at module level.

Data Processing. Functions of data processing mainly include remote measure processing program and remote signal processing program as shown in Fig 5. Instead of being deployed on dual host-backup, the program is deployed on multiple parallel data processing servers, each of which only has part of measuring records. The collection of measuring data in all data nodes constitutes full records. The programs only process data in the range of local measuring database. So it can reduce the pressure of data processing and improve efficiency of data processing greatly.

Formula Calculation. Formula calculation is different from the past in data accessing method as shown in Fig. 6. Formula calculation program is deployed on public node in charge of calculating all the 
formulas. Calculation parameters are read from corresponding data node by network interface. The program supports calculation of user-defined formulas.

Topology Coloring. Topology coloring is different from the past in data accessing method as shown in Fig. 7. Topology coloring program is deployed on public node in charge of topology analysis within supervisory control center. Calculation parameters are read from corresponding data node via network interface. The program completes analysis of the electrical island and implements the function of dynamic topology coloring according to dynamic topology connections and real-time data.

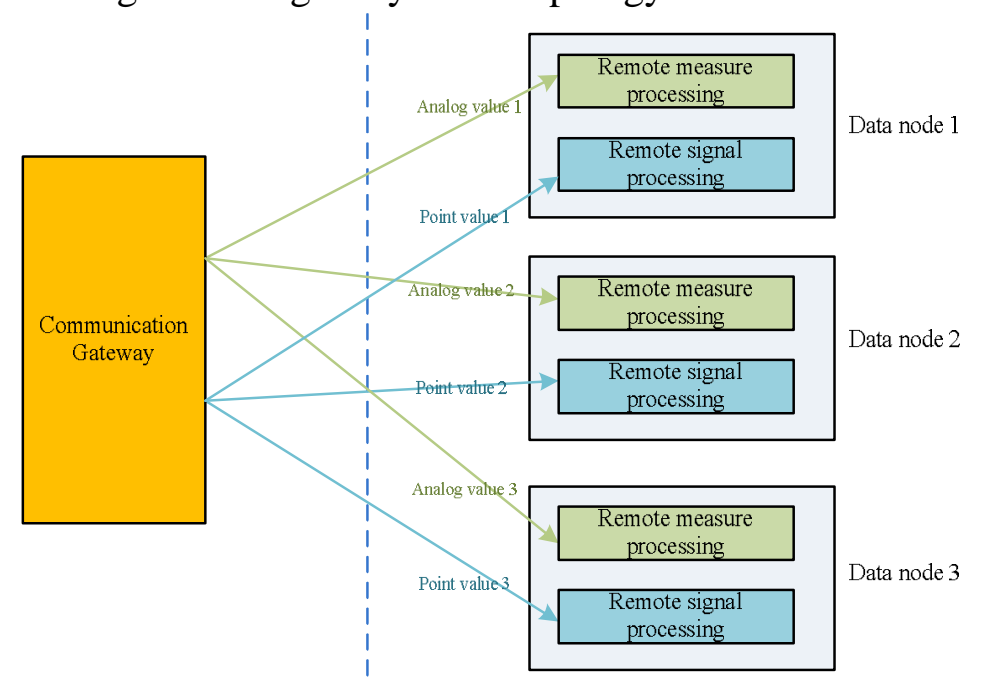

Fig. 5 Diagram of Data processing functions

\section{Wide Area Mutually Backup Mechanism in Distributed Supervisory Control System}

With the further development of smart grid, computer and communication technology has been widely applied in power grid. In order to establish resource-conserving construction mode of dispatching system, the new generation of dispatch technical supporting system makes reasonable data stream and service in system administration, system maintenance and equipment construction on the premise of full security [8]. It makes system information resource turn to "what needed is available" from "what needed does share", and it improves utilization efficiency of communication, network and hardware.

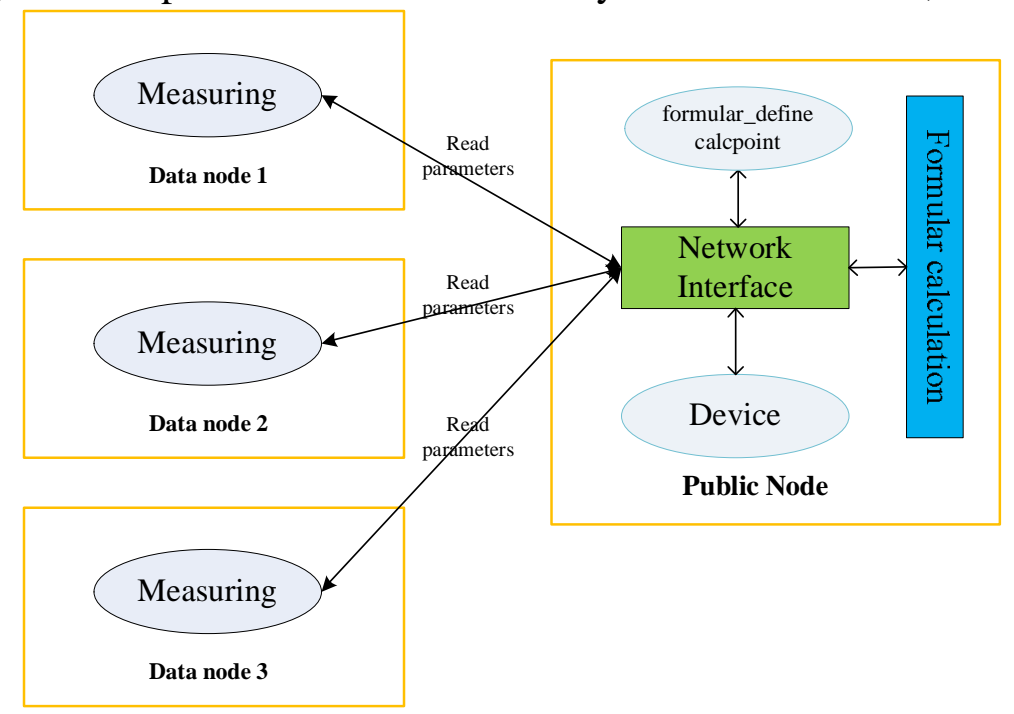

Fig. 6 Diagram of Formula calculation function

The new generation of distributed supervisory control system meets the basic requirements of communication, data network and model. It lays the foundation for wide area mutually backup. Firstly, the most important requirement on communication for new generation of system is the communication of grid data in WAN. Both the efficiency of data communication and the security of data in WAN should be guaranteed in this situation. Considering this new requirement, new wide area service bus 
can process and forward messages fast in cross-domain routing environment, and be compatible with all kinds of communication protocols, and support data encoding/decoding as needed. Secondly, the new generation of grid model center is the business center for basic information data storage, maintenance, release and management. It can meet the requirement of storage and maintenance of basic model data. On the other hand, it can support new business development in future based on new generation of architecture.

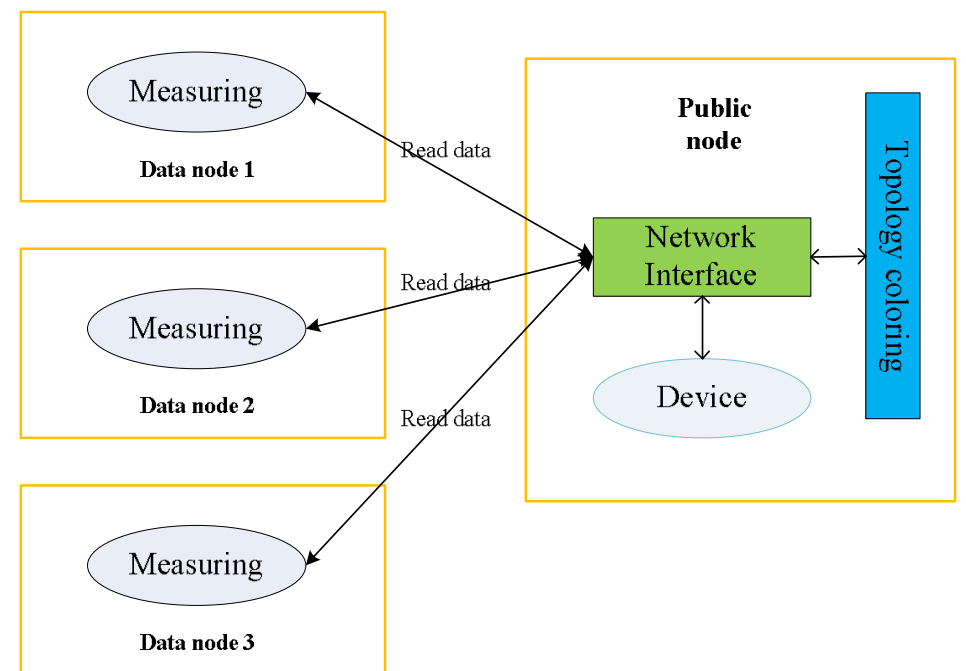

Fig. 7 Topology coloring Function

In new generation of system, model center provides model service for distributed supervisory control centers, and communication gateway sends real-time data to distributed supervisory control centers according to task assignment. The structure in normal operation is shown in Fig. 8.

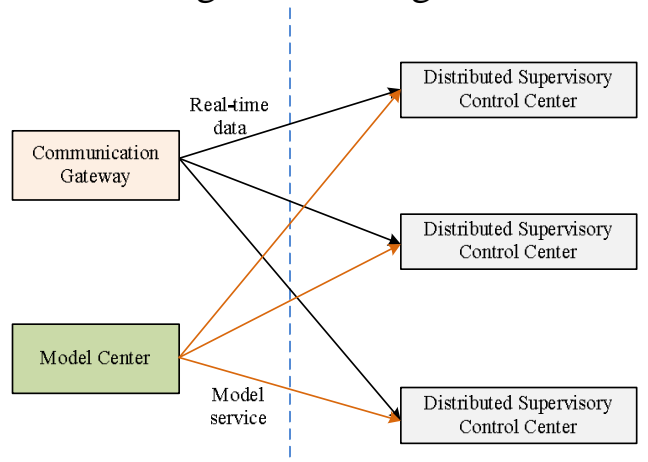

Fig. 8 System structure in normal operation

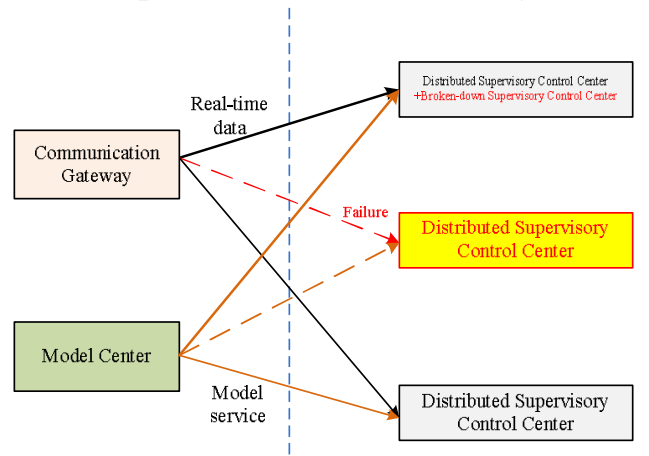

Fig. 9 Wide area mutually backup mechanism

When one of the distributed supervisory control centers breaks down, they can be mutually backup. Real-time data in broken-down center is assigned to SCADA message channel of other region by task assignment module. At the same time, model center releases the model of failure region to the newly-assigned center. Fig. 9 shows the mutually backup mechanism when failure happens.

Compared with traditional backup method, mutually backup in distributed supervisory control system has higher reliability, lower construction cost, less maintenance invest. The details are shown in Table 1: 
Table 1 Comparison between wide area mutually backup and traditional backup

\begin{tabular}{|c|c|c|c|}
\hline No. & $\begin{array}{c}\text { The technical and } \\
\text { economic indexes }\end{array}$ & Wide area mutually backup & Traditional backup \\
\hline 1 & Architecture & Wide area distributed & Active and standby \\
\hline 2 & System reliability & $\begin{array}{c}\text { Normal running when failure } \\
\text { happens }\end{array}$ & $\begin{array}{c}\text { System breaks down } \\
\text { when both host and } \\
\text { backup are broken-down }\end{array}$ \\
\hline 3 & $\begin{array}{c}\text { Reliability of data } \\
\text { acquisition }\end{array}$ & $\begin{array}{c}\text { Wide area distributed } \\
\text { acquisition, high reliability, } \\
\text { automatic switching when } \\
\text { server is down }\end{array}$ & $\begin{array}{c}\text { System doesn't work } \\
\text { when server is down }\end{array}$ \\
\hline 4 & $\begin{array}{c}\text { Cost of system } \\
\text { construction }\end{array}$ & Save more than $30 \%$ & Higher \\
\hline 5 & $\begin{array}{c}\text { System maintenance } \\
\text { Integrated with host and } \\
\text { backup, no extra staff needed }\end{array}$ & $\begin{array}{c}\text { The backup is } \\
\text { independent, need more } \\
\text { staff and cost }\end{array}$ \\
\hline
\end{tabular}

\section{Conclusion}

Compared with traditional supervisory control system, distributed supervisory control system explains the idea of "physical distribution and logical integration" better. By using wide area distributed technology, it deploys different levels of supervisory control centers in WAN, including provincial supervisory control center and districted supervisory control center. In each center, local real-time grid data is provided for local platform and server to support local SCADA, AGC/AVC, thus realizing the functions of dispersed monitoring, operation and control. In addition, distributed supervisory control system has the characteristic of wide area mutually backup, which has higher reliability, lower construction cost, less maintenance investment.

Within distributed supervisory control center, clustering SCADA technology has been adopted. Different from host-backup mode, workload is much more balancing between SCADA servers, and data processing pressure is much lower. It enhances system capacity for data processing by using parallel computing, and improves intensive level, reliability and extendibility of dispatching automation system.

\section{Reference}

[1] Xin Yaozhong. Development trend of power system dispatching automation technique in 21st century[J]. Power System Technology, 2001, 25(12):1-10.

[2] Yao Jianguo, Yang Shengchun, Gao Zonghe, Yang Zhihong. Development trend prospects of power dispatching automation system[J]. Automation of Electric Power Systems, 2007, 31(13):7-11.

[3] Yao Jianguo, Yang Shengchun, Chan Maohua. Reflections on operation supporting system architecture for future interconnected power grid[J]. Automation of Electric Power Systems, 2013, 37(21):52-59.

[4] Zhai Mingyu, Wang Jin, Wu Qingxi, Jin Jing, Wei Na. Architecture and key technologies of wide-area distributed real-time database system for power dispatching automation system[J]. Automation of Electric Power Systems, 2013, 37(2):67-71. 
[5] Zheng Weimin, Shi Wei. High Performance Cluster Computing[M]. Xian: Xian university press, 1999.

[6] Li Guiming, Yu Guoyang, Luo Jiarong. Establishment of Beowulf system based on linux[J]. Computer Engineering, 2003, 29(11):49-51.

[7] Sun Mingyang, Gao Yuan, Yan Yaqin, Meng Yongliang, Zhai Mingyu. Clustering technology for smart grid dispatching and control systems[J]. Automation of Electric Power Systems, 2015, 39(1):31-35.

[8] Wang Yimin, Xin Yaozhong, Xiang Li, Lu Changyan, Zou Guohui, Peng Qingqing. Security and protection of dispatching automation systems and digital networks[J]. Automation of Electric Power Systems, 2011, 25(21):5-8. 